\title{
DESIGN AND IMPLEMENTATION OF TIME EFFICIENT FLOATING POINT MULTIPLIER USING VHDL
}

Ameya Deshmukh ${ }^{1}$ and Pooja Hatwalne ${ }^{2}$

Abstract- This paper presents the design and implementation of time efficient single precision floating point multiplier using VHDL hardware description language. Comparison of various 8 bit fast adders is done using area and delay parameters and the efficient one is used in exponent addition of floating point multiplier. Similarly, comparison of two 24 bit multipliers is done on the area and delay parameters and the efficient one is used in mantissa multiplication of floating point multiplier. All these designs are synthesized and simulated in Xilinx ISE 14.7 targeted on xc3s50-5pq208 Spartan -3 device.

Keywords — single precision floating point multiplier, VHDL, fast adders, delay, Xilinx, Spartan-3.

\section{INTRODUCTION}

Floating point multipliers have variety of applications in fields like finance, medical imaging, biometrics, high performance audio etc. and also Multipliers play a key role in microprocessors, DSP processors and various FIR filters. A performance of a system is generally determined by the performance of the multiplier because the multiplier is generally the slowest element in the system. Since multiplication dominates the execution time of most DSP application so there is need of high speed multiplier. Hence increasing the speed and optimizing area of the multiplier is a major design issue.

This work deals with the design and implementation of optimized floating point multiplier. IEEE 754 single precision format is used to represent floating point numbers.

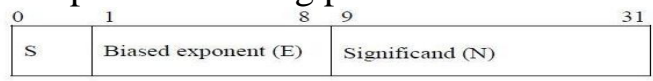

The general representation of floating point number is: (-1) $\wedge^{\wedge} \mathbf{S} * \mathbf{S} * \mathbf{1} \cdot \mathbf{1} * \mathbf{2}^{\wedge}(\mathbf{E}-\mathbf{1 2 7})$, where the range of $\mathrm{E}$ is -126 to +127 .

\section{Implementation Of Floating Point Multiplier}

A. Block diagram:

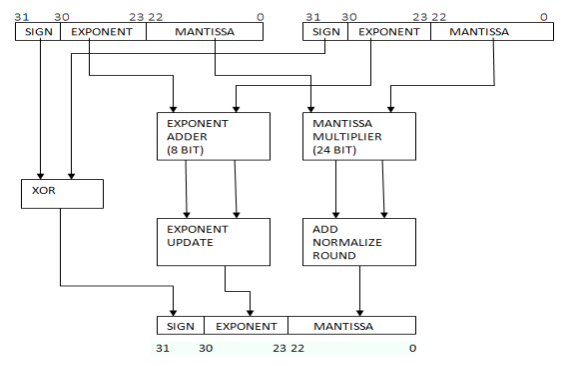

${ }^{1}$ Department of ECE, RCOEM Nagpur, $M H$

${ }^{2}$ Department of ECE, RCOEM Nagpur, $M H$ 
B. Algorithm of floating point multiplication with example:

The example below explains the algorithm of floating point multiplier properly:

Multiply 189.1234 by $-4.62=-873.750108$

First convert both of the floating point numbers to their equivalent IEEE754 formats:

- $\mathrm{A}=01000011001111010001111110010111=0 x 433 \mathrm{~d} 1 \mathrm{f} 97$

- $\mathrm{B}=11000000100100111101011100001010=0 \mathrm{xC} 093 \mathrm{~d} 70 \mathrm{a}$

\section{Step 1: Sign calculation:}

The sign is calculated by xor-ing the sign bits s1 and s2 of individual floating point numbers

$$
\begin{aligned}
\mathrm{S} & =\mathrm{s} 1 \text { XOR } \mathrm{s} 2 \\
& =0 \text { XOR } 1 \\
\mathrm{~S} & =1 .
\end{aligned}
$$

Step 2: Exponent calculation:

for exponent calculation bias is added to individual exponents E1 and E2 and it is to be removed after adding exponents to obtain the final exponent

$$
\begin{aligned}
& \text { E_output }=\mathrm{E} 1+\mathrm{E} 2-127 \\
& \text { Here } \mathrm{E} 1=10000110, \mathrm{E} 2=10000001 \\
& \text { E_output }=\mathrm{E} 1+\mathrm{E} 2-127=10000110+10000001-10000001=10001000
\end{aligned}
$$

Step3: Mantissa calculation:

Mantissa is of 23 bits but an implicit one is added for normalization so it becomes of 24 bits

For mantissa calculation the, individual mantissa M1 and M2 are added to get final mantissa of 48 bits.

Here we get: M_output $=1 . M 1 * 1 . M 2=1001011110010110000001000000000110011100110$

\section{Step 4: Normalization:}

In the output of the mantissa product the leading one comes in 46th position. As mentioned in the flow chart if the leading 1 comes in 46th position then consider the exponential part calculated in step 2 and take the bit position [45:23] which is calculated in step 3.

To get final output now we combine sign, exponent and normalized mantissa calculation:

$11000100010110100111000000000010=-873.750108$

\section{Proposed Floating Point Multiplier:}

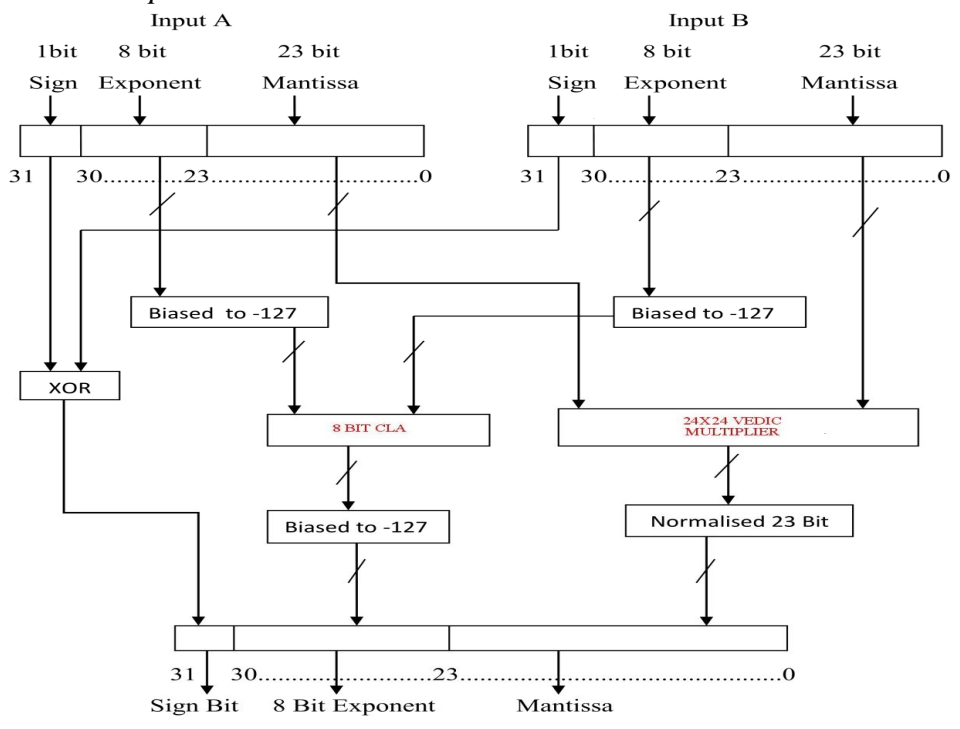

\section{Adders \& Their Performance Analysis}

Comparison of 4 fast adders namely, Ripple Carry Adder, Carry Look Ahead Adder, Carry Select Adder and Carry Save Adder is done on the area and delay parameters. Similarly comparison of Array Multiplier and Vedic Multiplier is done on the same parameters. For analysing area, Number of 4 input LUTs factor is considered whereas in case of delay, total of logic and route delay is used. 
A. Ripple Carry Adder

Ripple Carry Adder (RCA) is a basic adder which works on basic simple addition principle.RCA consists of series structure of Full Adders (FA). Each FA is used to add two bit along with carry bit. The carry generated from each full adder is given to next full adder and so on. Hence, the carry is propagated in a serial computation.

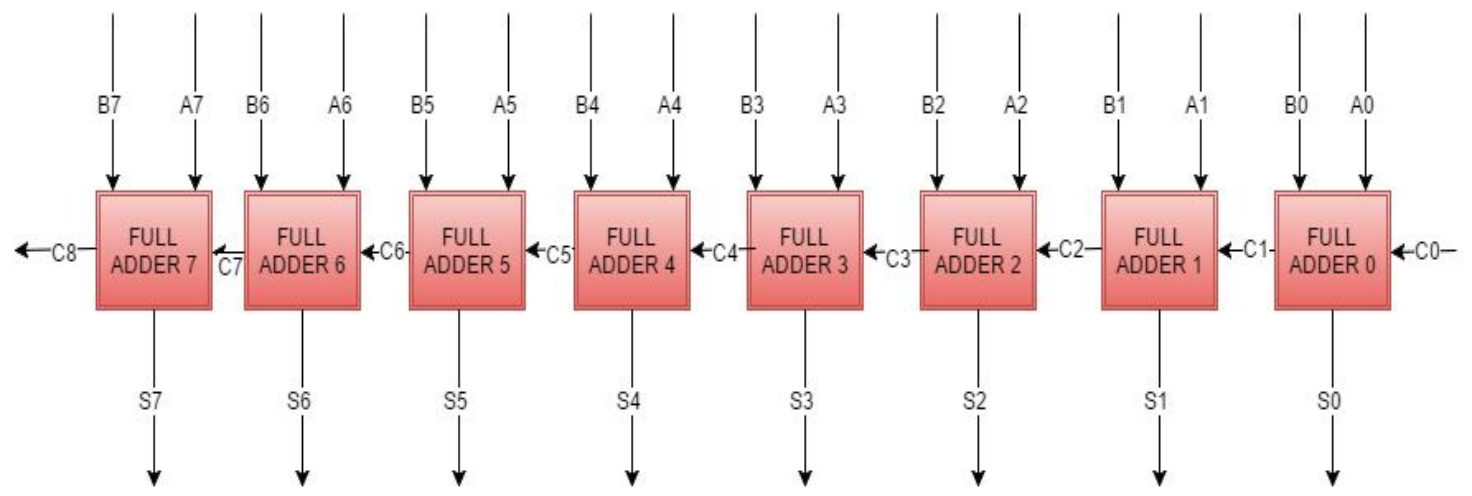

\section{B. Carry Look Ahead Adder}

For the Purpose of carry Propagation, Carry look Ahead Adder constructs Partial Full Adder, Propagation and generation Carry block. It avoids Carry propagation through each adder. In order to implement Carry Look Ahead Adder, first implement Partial Full Adder and then carry logic using propagation and generation block.

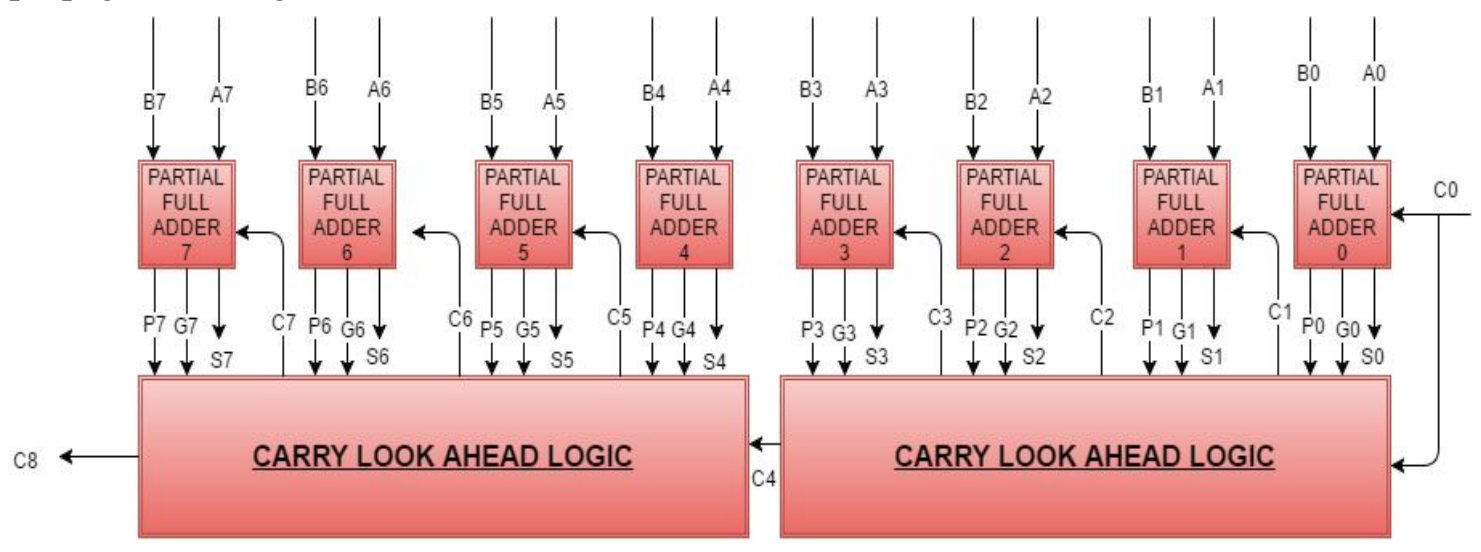

\section{Carry Select Adder}

Carry Select Adder can be constructed by implementing 2 stage Ripple Carry Adder and multiplexer circuit. Carry Select Adder select the sum and carry output from stage 1 ripple carry adder when carry input '0' and select Sum and carry output from stage 2 ripple carry adder, when carry input ' 1 '.For the purpose of selecting sum and carry output, $\mathrm{N}+1$ Multiplexer is implemented for $\mathrm{N}$ bit Addition Operation. 


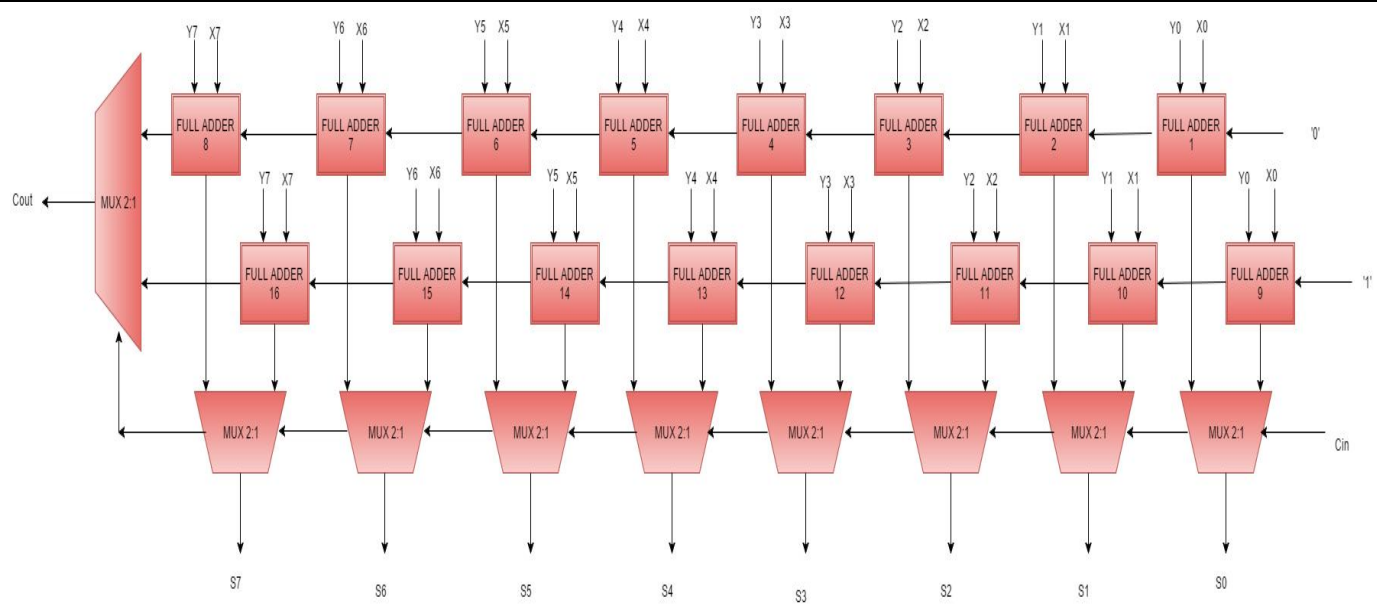

D. Carry Save Adder

In Carry Save Adder (CSA), three bits are added parallel at a time. In this scheme, the carry is not propagated through the stages. Instead, carry is stored in present stage, and updated as addend value in the next stage. Hence, the delay due to the carry is reduced in this scheme.

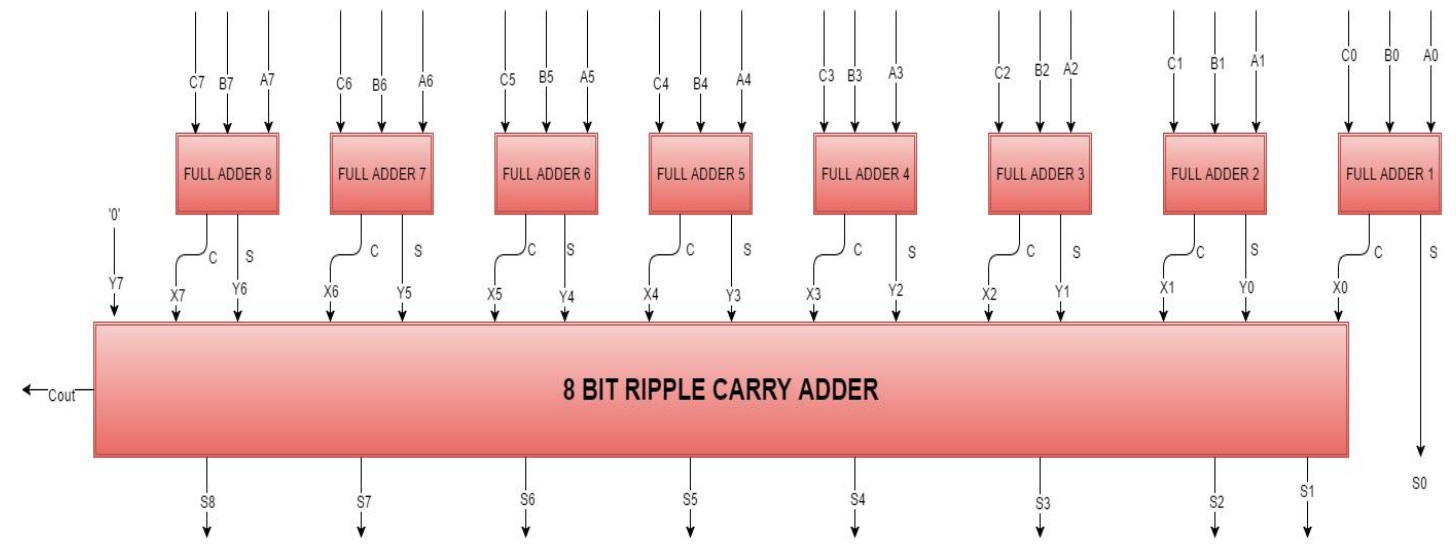

E. Performance Analysis of Adders

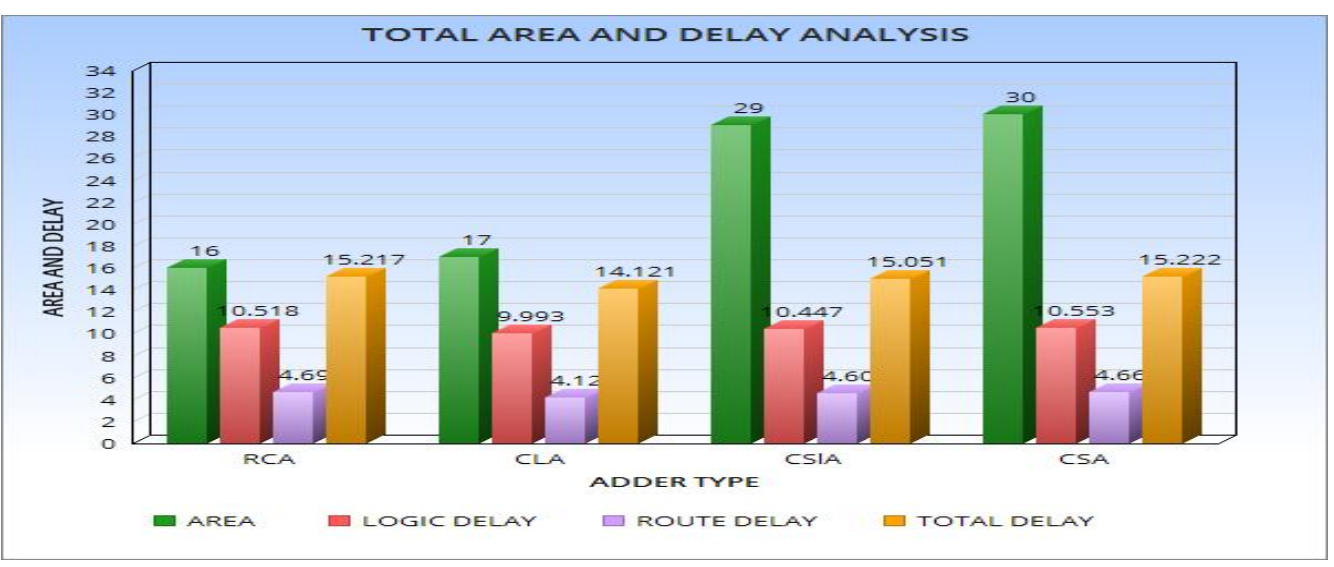


F. Conclusion

From total delay and area of all the adders, carry look ahead adder is found to be the most efficient adder and it is used in exponent addition of floating point multiplier.

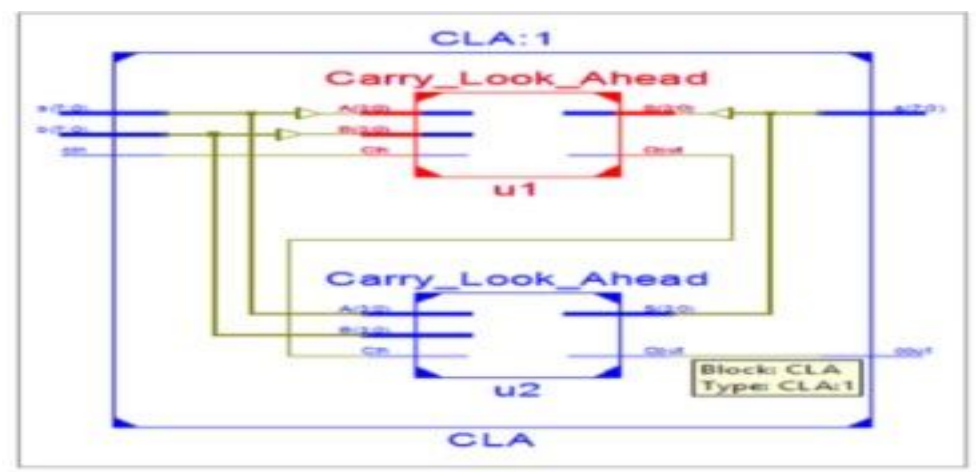

Fig 1. RTL VIew of Carry Look Ahead Adder
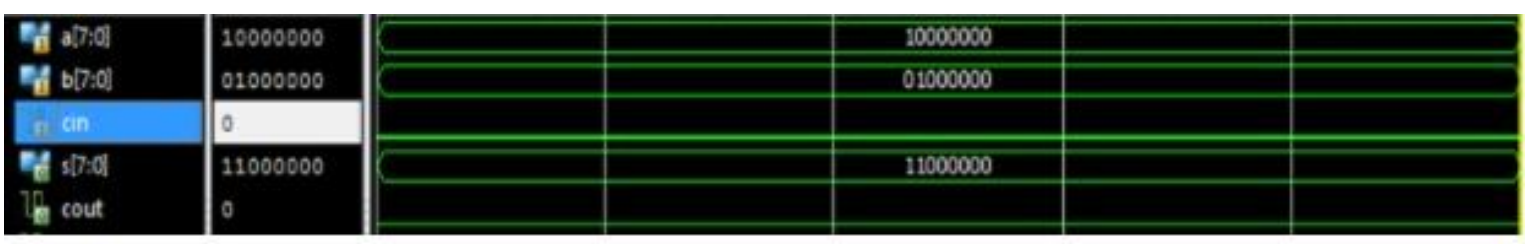

Fig 2. Simulation of Carry Look Ahead Adder

\section{Multipliers \& Their Analysis}

$24 \times 24$ Array Multiplier and 24x24 Vedic Multiplier is designed and analysed and the most delay efficient multiplier is chosen for floating point multiplier.

\section{A. Array Multiplier}

Array Multiplier is one of the best known and simplest multipliers. The multiplier is based on the shift and add algorithm. The partial products are generated by multiplying the multiplicand with one bit of the multiplier. The partial product is then shifted to the left in the order of the multiplier bit and then the partial products are added to give the final result. Figure below shows the basic principle of the 4X4 array multiplier. Here the LSB of the first partial product becomes the LSB of the product and the rest of the bits of the partial product is added to the second shifted partial product. The LSB of the addition becomes the next Bit of the product. This process is followed till the last partial product is added to the sum of the previous addition and the result of this addition is then placed in the product.

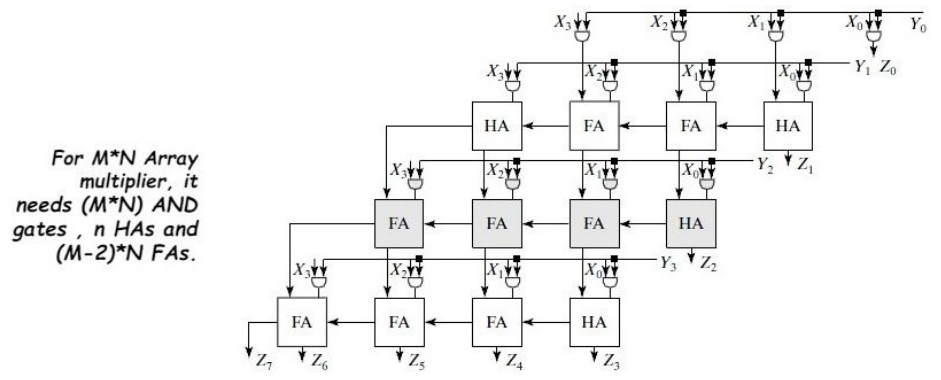


In Vedic Mathematics, Urdhva-Tiryakbhyam Sutra is a multiplication algorithm which is applicable to all cases of multiplication. Thus the multiplier will require the same amount of time to calculate the product and hence is independent of the clock frequency. In this method the partial products are generated simultaneously which itself reduces delay and makes this method fast.
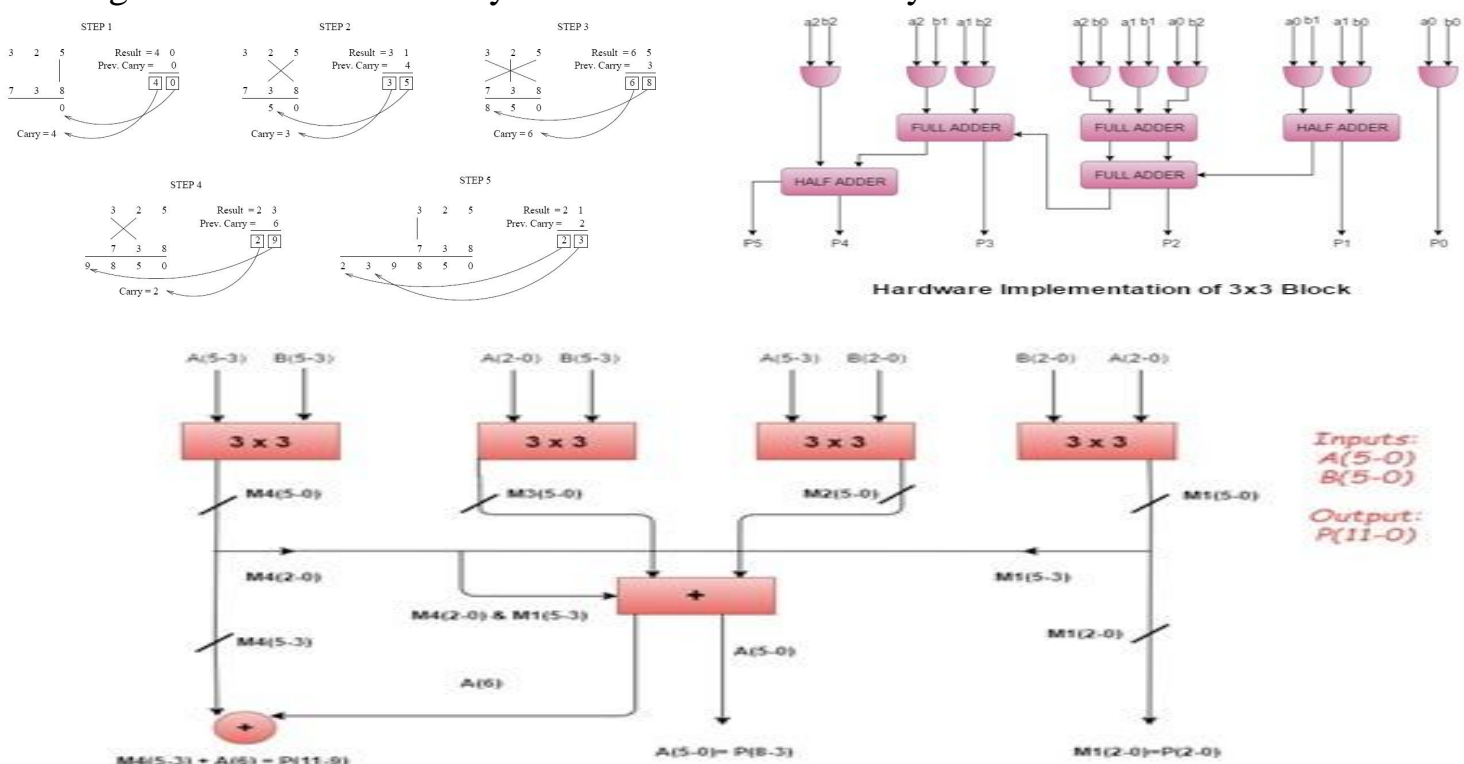

Hardware Implementation of $3 \times 3$ Block
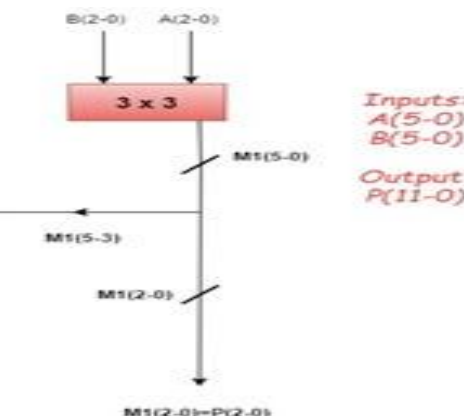

Hardware Implementation of $6 \times 6$ from $3 \times 3$ Block

For preparing a $24 \times 24$ multiplier, initially $3 \times 3$ block is made(as shown in fig1 above).Using it, $6 \times 6$ block is generated(as shown in fig 2 above)which is used to design a $12 \times 12$ block and then a $24 \times 24$ block is finally obtained using $12 \times 12$.

\section{Delay Analysis of Multipliers:}

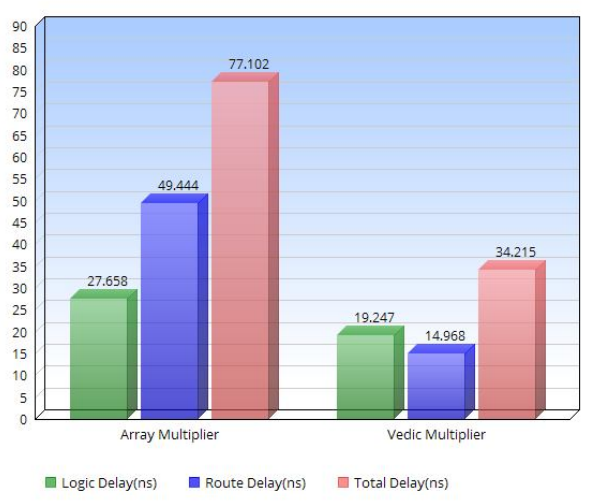

Clearly, Vedic Multiplier outshines Array Multiplier in every delay parameter thus giving superior timing performance and hence it is used in Mantissa calculation of floating point multiplier.

\section{Synthesis, Simulation \& Timing Performance Of Floating Point Multiplier}

The proposed Floating Point Multiplier is synthesized in Xilinx ISE 14.7 and simulated in Xilinx Isim Simulator and the RTL Schematic and Simulation results are shown below: 

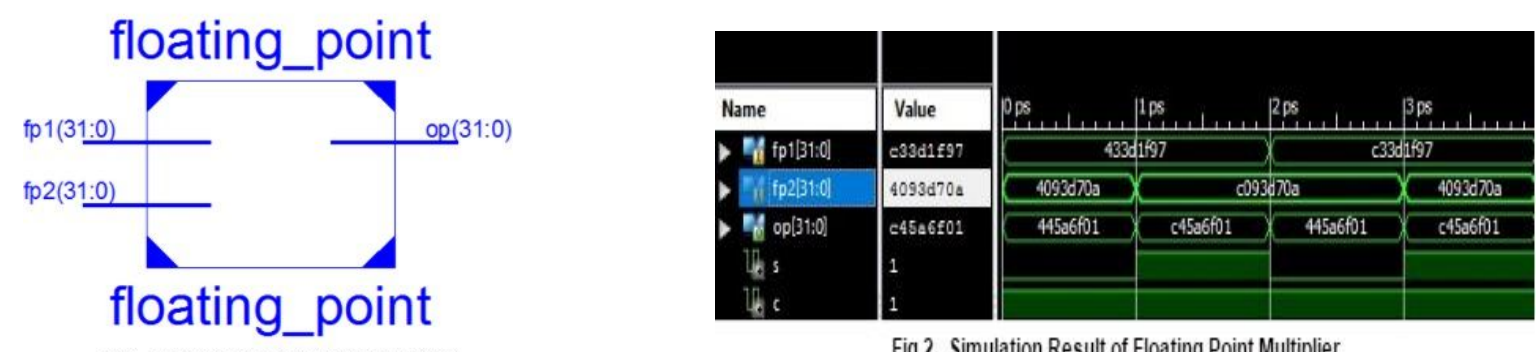

Fig 1. RTL Schematic of Floating Point Multiplier

Fig 2. Simulation Result of Floating Point Multiplier
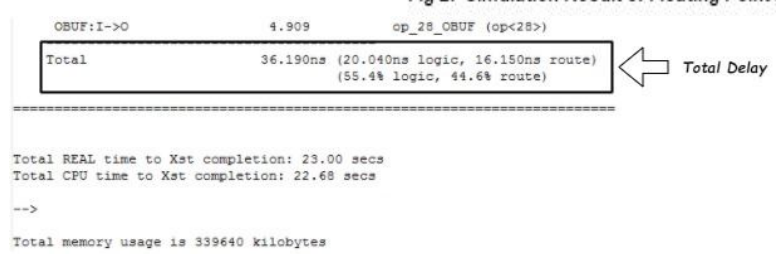

Fig 3. Delay of Floating Point Multiplier

\section{CONClusion}

This paper shows the implementation of IEEE754 single precision floating point multiplier on FPGA Spartan 3 using carry look ahead adder for exponent calculation and a Vedic multiplier for mantissa calculation. The proposed floating point multiplier shows optimized and better timing performance with a good accuracy. The results calculated are faster with total delay of 36.19ns. Due to an increasing demand of multipliers in scientific applications there is a need for increased precision floating point calculations using 64 bits and always there is a scope for improving the speed with the fast multiplication techniques and minimal time delay. So further the proposed work can be extended for the reconfigurable architecture and Double Precision Floating Point Multiplication

\section{REFERENCES}

[1] Paldurai.K, Dr.K.Hariharan, "FPGA Implementation of Delay Optimized Single Precision Floating point Multiplier" 2015 International Conference on Advanced Computing and Communication Systems (ICACCS -2015), Jan. 05 - 07, 2015, Coimbatore, INDIA.

[2]Mr. S.S.Mohanasundaram, A.Nirmal kumar, T.Arul prakash, "Design of Floating Point Multiplier Using Vedic Mathematics", IJISET - International Journal of Innovative Science, Engineering \& Technology, Vol. 2 Issue 1, January 2015.

[3]Bhavesh Sharma , Amit Bakshi, "Comparison of 24X24 Bit Multipliers for Various Performance Parameters" International Journal of Research in Advent Technology (E-ISSN: 2321-9637) Special Issue 1st International Conference on Advent Trends in Engineering, Science and Technology "ICATEST 2015”, 08 March 2015.

[4]Sneha Khobragade, Mayur Dhait, "Review on Floating Point Multiplier Using Vedic Mathematics" International Journal of Science and Research (IJSR) ISSN (Online): 2319-7064.

[5] C. Renard, "FPGA implementation of an IEEE Standard floating-point unit," Tech. Rep. Thayer School of Engineering, Dartmouth College, NH USA.

[6] I.V.Vaibhav, K.V.Saicharan, B.Sravanthi and D.Srinivasulu, "VHDL Implementation of Floating Point Multiplier using Vedic Mathematics", International Conference on Electrical, Electronics and Communications (ICEEC), ISBN978-93-81693-66-03 , June 2014 pp.110-115. 